\title{
BREVE RESEÑA SOBRE EL ULTRASONIDO TERAPÉUTICO
}

\section{A BRIEF REVIEW ON THERAPEUTHIC ULTRASOUND}

Marcos ALMIRÓN ${ }^{1}$.

${ }^{1}$ Universidad Nacional de Asunción, Facultad de Ciencias Médicas, San Lorenzo, Paraguay.

Cómo citar este artículo: Almirón M. Breve reseña sobre el ultrasonido terapéutico. Med. clín. soc. 2019;3(2):62-67.

\section{RESUMEN}

El ultrasonido es conocido por sus aplicaciones de baja intensidad en el diagnóstico médico. Sin embargo, el ultrasonido aplicado a mediana intensidad puede ser utilizado en la terapéutica de tejidos. En este artículo se hace una revisión narrativa, no sistemática, de la historia del desarrollo del ultrasonido, su técnica de aplicación, los efectos térmicos y no térmicos del mismo, las contraindicaciones de su utilización, así como las patologías o afecciones en las que su uso cuenta con evidencia.

Palabras clave: Ultrasonido; ultrasonido terapéutico; patologías.

\section{ABSTRACT}

Ultrasound is known for its low intensity applications in medical diagnosis. However, ultrasound applied at medium intensity can be used in tissue therapy. This article makes a narrative, non-systematic review of the history of ultrasound development, its application technique, its thermal and non-thermal effects, the contraindications of its use, as well as the diseases or conditions in which its use has evidence.

Keywords: Ultrasound; therapeutic ultrasound; diseases.

\section{HISTORIA DEL ULTRASONIDO}

A partir del siglo XVIII se hace notar el ultrasonido (US), como un fenómeno de la naturaleza cuando el biólogo italiano, Lazzaro Spallanzani descubre en el año 1700 la existencia de estas ondas, observando cómo los murciélagos atrapaban sus presas. En la primera mitad del siglo XIX (1803-1853), el físico y matemático austriaco Christian Andreas Doppler presenta su trabajo sobre el "Efecto Doppler" observando ciertas propiedades de la luz en movimiento, que eran aplicables a las ondas del US. Sobre la base de este estudio los japoneses cien años más tarde desarrollarían lo que hoy se conoce como la utilización del "Efecto Doppler" en US (1).

En la segunda mitad del siglo XIX los hermanos Pierre y Jacques Curie descubrieron las propiedades de algunos cristales conocidas como "Efecto piezoeléctrico", siendo la pieza clave y fundamental para el Desarrollo de los haces ultrasónicos. A comienzos del siglo XX, se realiza una de las primeras aplicaciones en el área de la marina, después de que el físico francés Paul Langevin inventara el Sonar, en el cual se basó el posterior desarrollo de los equipos usados 
en la aviación y luego en medicina terapéutica y diagnóstica. Terminada la segunda Guerra Mundial comienza el desarrollo de equipos diagnósticos en medicina, cuando grupos de investigadores japoneses, americanos y de algunos países europeos trabajan paralelamente para fabricar los primeros prototipos de equipos para diagnóstico médico en modo A (Analogue), y posteriormente en modo $B$ (Bright) con imagen analógica (1).

Luego de varios años de desarrollo, en la década de 1950, el US es aceptado por las sociedades médicas como instrumento de diagnóstico en medicina, dando origen a un sinnúmero de trabajos de investigación en distintas áreas de aplicación. El primer artículo publicado en una revista científica de prestigio, fue en Lancet, en 1958, donde se describió la experiencia en un grupo de 100 pacientes normales y con patología abdominal. La ciencia ha evolucionado desde entonces y hoy dia no solo vemos la utilizacion del US en el ambito de la medicina diagnostica, Tambien como uso terapeutico en rehabilitación fisica (1).

El ultrasonido médico es conocido por sus aplicaciones de baja intensidad en el diagnóstico médico, sin embargo, existen aplicaciones donde se usa ultrasonido en mediana intensidad en la terapéutica de tejidos. Estos equipos deben trabajar en un intervalo de intensidad segura para evitar efectos indeseables en el paciente; cuando se conocen las características ultrasónicas del campo acústico del transductor se está en la capacidad de prevenir estos efectos. En este trabajo se presenta el método C-scan con microsonda usado para obtener el campo acústico de un transductor ultrasónico de fisioterapia y así determinar la seguridad del tratamiento (2).

La terapia que usa la energía ultrasónica para producir cambios en los tejidos mediante sus efectos mecánicos y térmicos recibe el nombre de ultrasonido terapéutico. Para tratar cada padecimiento y debido a esta variedad de usos, se crearon diferentes protocolos que difieren, entre otras cosas, en la frecuencia, la potencia y el ciclo de trabajo. En un equipo US normal las frecuencias se pueden encontrar de $700 \mathrm{kHz}$ a $3 \mathrm{MHz}$ las cuales están limitadas por la profundidad de penetración en los tejidos. En general, por seguridad, la potencia de trabajo no debe ser mayor que $3 \mathrm{~W} / \mathrm{cm} 2$ (según la Organización Mundial de la Salud), y la Razón de Nouniformidad del Haz (BNR) debe estar entre dos y seis. El ciclo de trabajo indica el tiempo en que el transductor emite periódicamente; esta magnitud se mide en porcentaje y los tiempos de emisión y de no-emisión están en el orden de milisegundos (2).

Diferentes tipos de artefactos fueron construidos desde que se descubrieron los efectos de la energía ultrasónica sobre los tejidos. Éstos utilizan el ultrasonido para reparar tejidos, pero cada uno tiene sus propias características: transductores, sensores y protocolos de tratamiento (2).

El C-scan con microsonda es una de las técnicas utilizadas para la caracterización de transductores ultrasónicos. Este nombre se originó debido a que utiliza un elemento sensor pequeño para medir puntualmente los valores de intensidades acústicas (o algún otro parámetro relacionado) a lo largo del haz ultrasónico. Con este método es posible determinar la mayoría de los parámetros característicos de los transductores, tales como: el Área de Radiación Efectiva (ERA, Effective Radiating Area), la Razón de No-uniformidad del Haz (BNR, Beam Non-uniformity Ratio), el foco, el ángulo de divergencia, etc. 
Todos los tejidos presentarán una impedancia (resistencia aparente) al paso de las ondas sonoras. La impedancia específica de un tejido se determinará por su densidad y elasticidad. Para que la transmisión de energía sea máxima, la impedancia de los dos medios debe ser lo más parecida posible. Claramente en el caso del ultrasonido terapéutico cuya energía debe pasar de la máquina hacia diferentes tejidos, esta igualdad de impedancia es difícil de lograr.

Por lo tanto, cuanto mayor sea la diferencia de impedancia mayor será la divergencia que se producirá, y, por lo tanto, la energía transferida será menor. La diferencia de impedancia es mayor para la interfaz acero/aire, que es la primera que el ultrasonido terapéutico tiene que superar para alcanzar los tejidos.

Para minimizar esta diferencia se tiene que utilizar un medio de acoplamiento adecuado. Los medios de acoplamiento utilizados en este contexto incluyen agua, diversos aceites, cremas y geles. Idealmente, estos medios de acoplamiento deben tener las siguientes características: ser fluidos para llenar todos los espacios disponibles, ser relativamente viscosos para que permanezcan en su lugar, poseer una impedancia apropiada para los medios que conecta y deben permitir la transmisión del ultrasonido con una mínima absorción, atenuación o perturbación. En la actualidad, los medios basados en gel son preferibles a los aceites y cremas. El agua es un medio eficaz y puede utilizarse como alternativa, pero claramente no cumple los criterios anteriormente mencionados en términos de su viscosidad.

La absorción de energía del ultrasonido terapéutico sigue un patrón exponencial, es decir, los tejidos superficiales absorben más energía que los tejidos profundos. Para que la energía tenga un efecto debe ser absorbida, por lo tanto, esto debe ser considerado en relación con las dosificaciones del ultrasonido para lograr ciertos efectos.

Según Hoogland, la energía del ultrasonido terapéutico (dependiendo del tejido) alcanza aproximadamente las siguientes profundidades (3):

- $\quad$ Tejido muscular: $1 \mathrm{MHz}=9 \mathrm{~mm} ; 3 \mathrm{MHz}=3 \mathrm{~mm}$

- $\quad$ Tejido graso: $1 \mathrm{MHz}=50 \mathrm{~mm} ; 3 \mathrm{MHz}=16,5 \mathrm{~mm}$

- $\quad$ Tendón: $1 \mathrm{MHz}=6,2 \mathrm{~mm} ; 3 \mathrm{MHz}=2 \mathrm{~mm}$

Como es difícil, si no imposible, conocer el grosor de cada una de estas capas en cada uno de los pacientes, se emplean profundidades promedias para cada frecuencia: $1 \mathrm{MHz}=4 \mathrm{~cm} ; 3$ $\mathrm{MHz}=2 \mathrm{~cm}$. Sin embargo, algunas investigaciones sugieren que, en el entorno clínico, la energía del ultrasonido terapéutico puede alcanzar profundidades significativamente menores (3).

Como la penetración (o transmisión) del ultrasonido no es la misma en cada tipo de tejido, es evidente que algunos tejidos son capaces de absorber la energía en mayor proporción que otros. Generalmente, los tejidos con alto contenido de proteínas absorben la energía del ultrasonido en mayor medida, por lo tanto, los tejidos con alto contenido de agua y poca cantidad de proteínas absorben poca energía (por ejemplo, sangre y grasa), mientras que los tejidos con un alto contenido de proteínas y baja cantidad de agua absorberán la energía del ultrasonido más eficientemente (3).

En términos de práctica clínica, los tejidos que absorben más energía son aquellos con alto contenido de colágeno (ligamentos, tendones, fascia, cápsula articular, tejido cicatrizal). La aplicación del ultrasonido terapéutico a los tejidos con una baja capacidad de absorción de 
energía es menos probable que sea eficaz que la aplicación de la energía en un material de mayor absorción.

La mayoría de las máquinas de ultrasonido ofrecen la producción de energía de forma pulsada, y para muchos clínicos, este modo de tratamiento es preferible. Hasta hace poco, la duración del impulso (el tiempo durante el cual la máquina estaba encendida) era casi exclusivamente de $2 \mathrm{~ms}$ (milésimas de segundo) con un período de apagada variable. Ahora algunas máquinas ofrecen mayor variabilidad de estos tiempos (3).

Las típicas relaciones de pulso son $1: 1$ y 1:4, aunque hay otras disponibles. En el modo 1:1, la máquina ofrece una salida de 2 ms seguida de 2 ms de reposo. En el modo 1:4, la salida es de 2 ms seguida por un período de descanso de $8 \mathrm{~ms}$. Los efectos del ultrasonido pulsado están bien documentados, este tipo de modalidad es preferible especialmente en el tratamiento de las lesiones más agudas) (3).

\section{CONTRAINDICACIONES}

Como todo agente físico y técnica terapéutica, el ultrasonido también tiene sus contraindicaciones las cuales especifico a continuación (3):

- $\quad$ Durante el embarazo no aplique ultrasonido cerca del útero.

- $\quad$ No coloque ultrasonido sobre tejido canceroso.

- $\quad$ Evite aplicar ultrasonido en tejidos en fase de sangrado o en los cuales podría esperarse esta fase.

- No coloque ultrasonido sobre anomalías vasculares significativas incluyendo trombosis venosa profunda, embolia y arteriosclerosis severa.

- $\quad$ Pacientes con hemofilia.

- Aplicaciones sobre los ojos, sobre el ganglio cervicotorácico, en el área cardíaca en pacientes con enfermedad cardíaca avanzada o con marcapasos, en las gónadas o en las epífisis de crecimiento activas en los niños.

\section{INDICACIONES}

En la tabla 1 se observan las diferentes indicaciones $(4-17)$.

\begin{tabular}{|c|c|}
\hline \multicolumn{2}{|c|}{ TABLA 1. INDICACIONES DE ULTRASONIDO } \\
\hline Artrtis reumatoide en manos & Efecto térmico, evidencia en mejoría de dolor \\
\hline $\begin{array}{l}\text { Dolor musculoesqueletico de espalda } \\
\text { baja }\end{array}$ & $\begin{array}{l}\text { no se observa eficacia del ultrasonido terapéutico en disminución de } \\
\text { dolor de espalda baja }\end{array}$ \\
\hline Cicatrización de heridas & Se observa evidencia en la curación de heridas por su efecto no térmico \\
\hline $\begin{array}{l}\text { Edema óseo y osteonecrosis en esta- } \\
\text { dio inicial }\end{array}$ & $\begin{array}{l}\text { Mejoría clínica y funcional por su efecto no térmico asociado a laserte- } \\
\text { rapia de mediana intensidad }\end{array}$ \\
\hline $\begin{array}{l}\text { Fracturas de huesos largos. Retardo } \\
\text { de consolidación }\end{array}$ & $\begin{array}{l}\text { Buena evolución en proceso de reparación ósea y disminución en Desa- } \\
\text { rrollo de complicaciones, mejoría utilizando el ultrasonido a modo pul- } \\
\text { sado }\end{array}$ \\
\hline Gonartrosis de II y III grado & $\begin{array}{l}\text { Solida evidencia de disminución de dolor por su modo pulsado y a baja } \\
\text { intensidad }\end{array}$ \\
\hline Osteoartritis de rodilla & $\begin{array}{l}\text { Efecto no térmico y a baja intensidad mejoría sintomatológica y funcio- } \\
\text { nalidad pero carece de evidencia científica. }\end{array}$ \\
\hline $\begin{array}{l}\text { Parálisis cerebral infantil; espasticidad } \\
\text { leve }\end{array}$ & $\begin{array}{l}\text { Ultrasonido terapéutico asociado a elongación muscular pasiva, expresa } \\
\text { mejoría clínica que solo las elongaciones pasivas por si solas }\end{array}$ \\
\hline
\end{tabular}




\begin{tabular}{|c|c|}
\hline Espasticidad leve en niños & $\begin{array}{l}\text { Efecto termino, de baja intensidad asociado a elongaciones musculares, } \\
\text { mejor método de tratamiento no invasiva para contracturas musculares } \\
\text { de origen neurológico. }\end{array}$ \\
\hline Prótesis cementada de cadera & $\begin{array}{l}\text { Favorece la consolidación y fijación del cemento de la prótesis al tejido } \\
\text { óseo. }\end{array}$ \\
\hline Síndrome subacromial & No existe evidencia del ultrasonido terapéutico frente al placebo \\
\hline Síndrome miofascial & $\begin{array}{l}\text { el efecto terapéutico; metabolismo y analgesia del ultrasonido muestra } \\
\text { efectividad por su modalidad térmica }\end{array}$ \\
\hline $\begin{array}{l}\text { Adiposidad localizada en región in- } \\
\text { fraumbilical }\end{array}$ & $\begin{array}{l}\text { Ultrasonido de alta potencia arroja resultados altamente significativos } \\
\text { en favor a su utilización como modalidad terapéutica no invasiva }\end{array}$ \\
\hline $\begin{array}{l}\text { Respuesta fisiológica del tejido con- } \\
\text { juntivo }\end{array}$ & $\begin{array}{c}\text { Efectos termales y no termales favorecen a la síntesis de colágeno sobre } \\
\text { todo en la estructura tendinosa. }\end{array}$ \\
\hline $\begin{array}{l}\text { Ulcera por presión en adulto mayor } \\
\text { con dependencia severa }\end{array}$ & $\begin{array}{l}\text { Ultrasonido terapéutico demostró mejoría porcentual en reparación de } \\
\text { tejido de aquellos pacientes que no recibieron pero no demostró dife- } \\
\text { rencia estadísticamente significativa }\end{array}$ \\
\hline Cervicalitas & $\begin{array}{l}\text { Ultrasonido terapéutico de modalidad continua y altas intensidad mos- } \\
\text { tro disminución de dolor en pacientes a diferencia de bajas intensida- } \\
\text { des. }\end{array}$ \\
\hline
\end{tabular}

\section{CONFLICTOS DE INTERÉS Y FUENTE DE FINANCIACIÓN}

El autor declara no poseer conflictos de interés. Fuente de financiación: ninguna.

\section{REFERENCIAS BIBLIOGRÁFICAS}

1. Ortega T, Seguel B S. Historia del ultrasonido: el caso chileno. Revista chilena de radiología. 2004;10(2):89-92. http://dx.doi.org/10.4067/S0717-93082004000200008

2. Gutiérrez MI. Determinación y Evaluación del Campo Acústico de Transductores UItrasónicos para Fisioterapia. En México; 2008. p. 6.

3. Todo sobre el ultrasonido terapéutico, aplicaciones, indicaciones y contraindicaciones [Internet]. [citado 28 de agosto de 2019]. Disponible en: https://www.fisioterapiaonline.com/articulos/todo-sobre-el-ultrasonido-terapeutico-aplicaciones-indicaciones-y-contraindicaciones

4. Villaseñor Moreno JC, Escobar Reyes VH, Andrade LP de la L, Ramírez BIG. Síndrome de dolor miofascial. Epidemiologia, fisiopatología, diagnóstico y tratamiento. Rev Esp Med Quir. 2013;18(2):148-57. URL.

5. Valladares-Bartolomé J. Edema óseo e incipiente osteonecrosis en cóndilo femoral externo tratados con láser y ultrasonido. A propósito de un caso. Fisioterapia. 2013;35(1):40-3. https://dx.doi.org/10.1016/i.ft.2012.09.001

6. Valentim da Silva RM, Froes Meyer P, Ranaco Santos B, de Oliveira Félix JL, Ronzio OA. Efectos del ultrasonido de alta potencia en la adiposidad localizada. Fisioterapia. 2015;37(2):55-9. https://dx.doi.org/10.1016/i.ft.2014.06.003

7. San Segundo R, Molins J, Valdés M, Fernández T. Tratamiento conservador del síndrome subacromial. Ultrasonidos frente a placebo. Un ensayo clínico. Rehabilitación (Madr). 2008;42(2):61-6. https://dx.doi.org/10.1016/S0048-7120(08)73615-6

8. Rúa ORR, Monreal R, Chong-Quero JE. Ultrasonido de baja intensidad para el tratamiento de fracturas óseas con retardo en la curación. Revista Mexicana de Ingeniería Biomédica. 2005;26(1):52-6. URL.

9. Pedraza Mejías C, Martínez Cañadas J. Respuesta fisiológica del tejido conjuntivo de músculos y tendones tras la aplicación de los agentes físicos. Fisioterapia. 2008;30(6):279-85. https://dx.doi.org/10.1016/i.ft.2008.09.013 
10. Nemegyei JÁ, Ojeda ZR, Zapata FÁ, Velázquez JD. Ultrasonido terapéutico versus fluidoterapia para la afección manual en artritis reumatoide. Un ensayo clínico controlado. Residente. 2010;5(1):33-9. URL.

11. Morales-Osorio MA, Torrado Navarro C. Dolor y Modalidades Físicas: un Nuevo Paradigma en Fisioterapia. Revista Científica Salud Uninorte. 2014;30(3):465-82. URL.

12. Mancilla-Solorza E, Bascuñán-Retamal S, Bravo-Garrido B, Ibarra-Ábalos N. Efectos del ultrasonido terapéutico en el tratamiento de las úlceras por presión en adultos mayores con dependencia severa. Rehabilitación (Madr). 2012;46(2):103-11. https://dx.doi.org/10.1016/j.rh.2012.02.002

13. Loyola Sanchez A, Ramirez Wakamatzu MA, Vazquez Zamudio J, Casasola J, Hernandez Cuevas C, Ramirez Gonzalez A, et al. Efecto del ultrasonido terapeutico pulsatil de baja intensidad sobre la regeneracion del cartilago articular en pacientes con gonartrosis de segundo y tercer grado. Reumatología Clínica. 2009;05(4):163-7. URL.

14. Cigarroa I, Palomera FA, Pino JP, Hernández HC, Sepúlveda SA, Soto CA, et al. Ganancia de extensión de rodilla usando ultrasonido terapéutico y estiramiento pasivo en pacientes con parálisis cerebral espástica leve. Fisioterapia. 2016;38(3 (Mayo-Junio 2016)):118-26. URL.

15. Arriaga Hernández PR, Herrera Marmolejo M, Parodi Carbajal AR. Efectividad de la aplicación de ultrasonido terapéutico y ejercicio de estiramiento a músculos isquiotibiales en niños con parálisis cerebral tipo diparesia espástica leve. Rehabilitación (Madr). 2010;44(4):326-30. https://dx.doi.org/10.1016/j.rh.2010.07.005

16. Alonso CA. Aplicación de ultrasonidos en el dolor cervical de origen inespecífico. Fisioterapia. 2007;29(4):176-82. URL.

17. Aguilar-Velázquez DG, Ávila RGC, Narvaez LL, Arias-Vázquez PI, Tovilla-Zárate CA. Eficacia del ultrasonido terapéutico en el dolor musculoesquelético de espalda baja: una revisión comprensiva. Revista Colombiana de Medicina Física y Rehabilitación. 2018;27(2):140-51. http://dx.doi.org/10.28957/rcmfr.v27n2a3 